\title{
Isochorismatase domain-containing protein 1 (ISOC1) participates in DNA damage repair and inflammation-related pathways to promote lung cancer development
}

\author{
Jinghan Shi ${ }^{1 \#}$, Fujun Yang ${ }^{1 \#}$, Nanfeng Zhou ${ }^{2 \#}$, Yan Jiang ${ }^{1}$, Yanfeng Zhao ${ }^{1}$, Junjie Zhu ${ }^{1}$, Arsela Prelaj ${ }^{3,4}$, \\ Jyoti Malhotra $^{5}$, Nicola Normanno ${ }^{6}$, Elisa Danese ${ }^{7}$, Andrés F. Cardona ${ }^{8}$, Xuan Hong ${ }^{9}$, Gening Jiang ${ }^{1}$, \\ Xiao Song ${ }^{1}$
}

${ }^{1}$ Department of Thoracic Surgery, Shanghai Pulmonary Hospital, Tongji University School of Medicine, Shanghai, China; ${ }^{2}$ Department of Radiation Oncology, Shanghai Pulmonary Hospital, Tongji University School of Medicine, Shanghai, China; ${ }^{3}$ Medical Oncology Department, Fondazione IRCCS Istituto Nazionale Tumori, Milan, Italy; ${ }^{4}$ Department of Electronics, Information, and Bioengineering, Polytechnic University of Milan, Milano, Italy; ${ }^{5}$ Rutgers Cancer Institute of New Jersey, New Brunswick, NJ, USA; ${ }^{6}$ Cell Biology and Biotherapy Unit, Istituto Nazionale TumoriIRCCS_-Fondazione G. Pascale", Naples, Italy; ${ }^{7}$ Section of Clinical Biochemistry, University of Verona, Verona, Italy; ${ }^{8}$ Foundation for Clinical and Applied Cancer Research-FICMAC/Clinical and Translational Oncology Group, Clínica del Country/Molecular Oncology and Biology Systems Research Group (Fox-G), El Bosque University, Bogotá, Colombia; ${ }^{9}$ Department of Thoracic Surgery, Shanghai East Hospital, Tongji University School of Medicine, Shanghai, China

Contributions: (I) Conception and design: Jinghan Shi, Xuan Hong, Gening Jiang, and Xiao Song; (II) Administrative support: Xuan Hong, Gening Jiang, and Xiao Song; (III) Provision of study materials or patients: Jinghan Shi, Fujun Yang, Yan Jiang, and Yanfeng Zhao; (IV) Collection and assembly of data: Jinghan Shi, Fujun Yang, Nanfeng Zhou, Junjie Zhu, and Xiao Song; (V) Data analysis and interpretation: All authors; (VI) Manuscript writing: All authors; (VII) Final approval of manuscript: All authors.

\#These authors contributed equally to this work.

Correspondence to: Xiao Song, PhD. Shanghai Pulmonary Hospital, Tongji University School of Medicine, 507 Zhengmin Road, Shanghai 200433, China. Email: songxiao198327@163.com; Gening Jiang, MD. Shanghai Pulmonary Hospital, Tongji University School of Medicine, 507 Zhengmin Road, Shanghai 200433, China. Email: jgnwp@aliyun.com; Xuan Hong, MD. Shanghai East Hospital, Tongji University School of Medicine, 150 Jimo Road, Shanghai 200120, China. Email: hxseh@126.com.

Background: The advent of novel molecular targets has dramatically changed the treatment landscape of lung cancer in recent years. Isochorismatase domain-containing protein 1 (ISOC1) has been reported as a potential biomarker in gastrointestinal cancer, while its function in lung cancer has not been determined.

Methods: The expression levels and prognostic significance of ISOC1 were assessed using bioinformatic analysis. Overexpression of ISOC1 and miR-4633, and knockdown of ISOC1 in non-small cell lung cancer (NSCLC) cell lines were generated by lentiviral infection with overexpressed or shRNA plasmids. CRISPR/ Cas9 system was applied to knockout ISOC1 in A549 cells. The functions of ISOC1 and miR-4633 in lung cancer development were investigated using cell proliferation, migration, and invasion assays. Xenograft tumor growth assays in nude mice were further assessed the effect of ISOC1 in the tumorigenesis of NSCLC in vivo. Cell cycle distribution analysis was performed to uncover the underlying mechanism of ISOC1 and miR-4633 in promoting NSCLC cell proliferation. Co-immunoprecipitation combined with mass spectrometry and RNA sequencing were performed to uncover the potential mechanism of ISOC1 in lung cancer development.

Results: Our results found that ISOC1 expression was upregulated in NSCLC tissues and that increased expression of ISOC1 was significantly associated with worse disease-free survival in NSCLC patients. Overexpression of ISOC1 could increase the proliferation, viability, migration, and invasion of NSCLC cells. Furthermore, miR-4633, located in the first intron of ISOC1, could also promote tumor cell progression and metastasis. Mice xenograft tumor assay showed that knockout of ISOC1 could significantly inhibit tumor growth in vivo. Besides, co-immunoprecipitation combined with mass spectrometry assay revealed that ISOC1 interacted with the proteins of DNA damage repair pathways and that upregulated ISOC1 expression 
could significantly increase the number of DNA damage lesions. RNA sequencing analysis showed that the downstream signaling pathways mediated by ISOC1 were mainly inflammation-related.

Conclusions: We demonstrated that ISOC1 and its intronic miR-4633, both of them could promote NSCLC cell proliferation, migration, invasion, and cell cycle progression. ISOC1 participates in DNA damage repair and inflammation to promote lung cancer development.

Keywords: Isochorismatase domain-containing protein 1 (ISOC1); miR-4633; lung cancer; DNA damage repair; inflammation

Submitted Dec 31, 2020. Accepted for publication Mar 25, 2021.

doi: $10.21037 /$ tlcr-21-219

View this article at: http://dx.doi.org/10.21037/tlcr-21-219

\section{Introduction}

Non-small cell lung cancer (NSCLC) accounts for $85 \%$ of all malignant lung tumors, with adenocarcinoma being the predominant histologic subtype of NSCLC (1). Novel treatment strategies based on targeted therapies and immunotherapies combined with platinum-based chemotherapy have produced improved survival benefits in NSCLC compared to traditional chemotherapy significantly prolonging the overall survival (OS) $(2,3)$. To improve lung cancer patients' prognosis, further efforts should be made to discover new molecular drivers and develop targeted therapies.

Isochorismatase domain-containing protein 1 (ISOC1), also known as CGI-111, belonging to the family of hydrolases, can catalyze the transformation of isochorismate into 2,3-dihydroxy-2,3-dihydrobenzoate and pyruvate, with another substrate-water (4). The human ISOC1 gene is located on chromosome $5 \mathrm{q} 23.3$ and encodes a protein of 298 amino acids. Previous studies have reported that ISOC1 is related to eye and testis development in animal models $(5,6)$. ISOC1 has been reported to be involved in neutrophil development regulated by microRNA-130A (miRNA130A) in mice and humans (7). In addition to the above functions, ISOC1 was also found to play important human tumor biology roles. For instance, ISOC1 was identified to be deregulated in uterine leiomyoma (8) and was also proven to act as an estrogen-responsive gene, promoting breast cancer cells' growth (9). Two recent studies revealed that knockdown of ISOC1 could impair tumorigenesis and gastrointestinal cancer progression through activating protein kinase B (Akt)/glycogen synthase kinase-3 beta (GSK-3 $\beta$ ) pathway $(10,11)$. However, the role of ISOC1 in lung carcinogenesis is still unknown.

MicroRNAs (miRNAs) commonly lead to messenger
RNA (mRNA) degradation and translation inhibition via binding to specific sequences. A number of miRNAs have been reported to be dysregulated in NSCLC and lead to the dysfunctions of oncogenic-related pathways (12). Intronic miRNAs, which are located on the intron of the host gene, can mediate and/or assist the host gene in performing various biological functions (13). The first intron of ISOC1 encodes intronic miR-4633, was reported to be involved in the lung fibrosis mediated by transforming growth factor beta (TGF- $\beta$ ) and to contribute to the antitumor activity in sinonasal mucosal melanoma $(14,15)$. Currently, limited knowledge on miR-4633 exists, and its function in NSCLC remains to be explored.

This study was a functional investigation of two lung cancer cell lines, A549 and H1299, with the aim to explore the roles of ISOC1 in the development and progression of NSCLC. Co-immunoprecipitation (Co-IP) combined with mass spectrometry (MS) and RNA sequencing were performed to uncover the potential mechanism of ISOC1 in lung cancer development.

We present the following article in accordance with the ARRIVE reporting checklist (available at http://dx.doi. org/10.21037/tlcr-21-219).

\section{Methods}

\section{Cell culture}

Human NSCLC cell lines A549 and NCI-H1299, and human embryonic kidney cell line, HEK-293/HEK-293T, were preserved in the lab of Thoracic Surgery of Shanghai Pulmonary Hospital. NSCLC cells were cultured in RPMI-1640 medium supplemented with $10 \%$ fetal bovine serum (FBS, Gibco, USA), $100 \mathrm{U} / \mathrm{mL}$ penicillin, and 100 mg/mL streptomycin (Gibco). HEK-293/HEK-293 T 
cells were grown in complete Dulbecco's Modified Eagle's Medium (DMEM) supplemented with 10\% FBS. All cells were maintained at $37^{\circ} \mathrm{C}$ in a humidified incubator with $5 \% \mathrm{CO}_{2}$.

\section{$R N A$ extraction and quantitative real-time polymerase chain reaction ( $q R T-P C R)$}

Total RNA was extracted from cultured cells which were harvested with TRIzol reagent (Invitrogen, USA) to quantify the expression of relevant genes. The reverse transcription reactions were carried out according to the manufacturer's protocol of commercially available kits (Vazyme, China). qRT-PCR was performed on a LightCycler 96 (Roche, Switzerland) and detected with THUNDERBIRD SYBR qPCR Mix (Toyobo, Japan). The primer sequences used in this study are listed in Table S1. The qPCR conditions were $95^{\circ} \mathrm{C}$ for $3 \mathrm{~min}$, and then 40 cycles at $95^{\circ} \mathrm{C}$ for $15 \mathrm{~s}$ and $60^{\circ} \mathrm{C}$ for $45 \mathrm{~s}$. The differences in mRNA expression were compared using the comparative cycle threshold (Ct) method. Beta-actin was detected as the endogenous control for ISOC1.

\section{Plasmid construction and lentivirus infection}

To generate ISOC1 (ISOC1 OE) and miR-4633 stably overexpressed NSCLC cell lines, the full-length of ISOC1 and miR-4633 were amplified from complementary DNA (cDNA) and genomic DNA (gDNA) from the NSCLC cell line A549 by PCR and were cloned into the pCDHCMV-MCS-EF1-GFP+Puro (CD513B) plasmid (System Biosciences, USA). To stably interfere with ISOC1 expression, the short hairpin RNA (shRNA) sequences targeting ISOC1 were designed and cloned into the plasmid LentiLox 3.7 (pLL3.7) to knock down the expression of ISOC1 (ISOC1 KD). The CD513B empty vector (EV) and pLL3.7 EV were used as controls for CD513B-ISOC1 and pLL3.7-shISOC1 (shISOC1-1 and shISOC1-2), respectively. Lentiviral production, collection, and infection were performed as described in previous studies (16).

To silence ISOC1 expression at DNA level, clustered regularly interspaced short palindromic repeats and an associated protein-9 nuclease (CRISPR/Cas9) system was applied to knockout ISOC1 (ISOC1 KO) in A549 cells. To construct a single Cas-ISOC1-single guide RNA (sgRNA) vector, ISOC1 sgRNA was designed using the sgRNA design website (http://crispr.mit.edu/). The construction of the plasmid was conducted as previously reported (17).

\section{Dual-luciferase reporter assay}

The 3'-untranslated region (3'UTR) of ISOC1 was cloned into the pGL3-promoter luciferase reporter plasmid (Invitrogen). HEK-293 cells were cultured in 24-well plates and transfected with $0.5 \mu \mathrm{g}$ of pGL3-promoter constructs containing 3'UTR of ISOC1 or EV together with $0.3 \mu \mathrm{g}$ CD513B-miR-4633 or EV plasmids using Lipofectamine 2000 (Invitrogen) as the transfection reagent. After 36h, the cell lysate was collected to determine the firefly and renilla luciferase signals using the dual-luciferase reporter assay system (Promega, USA).

\section{Western blot analysis}

Transfected cells were lysed and total proteins were extracted using cell lysis buffer for Western blot and immunoprecipitation (Beyotime Biotechnology, China). Equal amounts of proteins were separated by $10-12 \%$ SDS-PAGE and were then transferred onto polyvinylidene difluoride (PVDF) membranes (Millipore, USA). The membranes were blocked with tris-buffered saline (TBS) solutions (containing $5 \%$ fat-free milk powder and $10 \%$ Tween 20) at room temperature for $1 \mathrm{~h}$ and incubated with ISOC1 (1:1,000 dilution; Abcam, USA) or beta-actin (1:2,000 dilution; Abcam, USA) overnight at $4{ }^{\circ} \mathrm{C}$, followed with secondary antibody (1:5,000 dilution; Cell Signaling Technology, USA) at room temperature for $2 \mathrm{~h}$. The blots were visualized using enhanced chemiluminescence (ECL) solutions and an imaging system (GE Healthcare, USA).

\section{Cell proliferation assay}

Cell Counting Kit-8 (CCK-8, Dojindo, Japan) assay was carried out to evaluate cell proliferation in different NSCLC cell lines, including the ISOC1 OE, KD, and KO groups. Approximately 1,500 cells/well were seeded into a 96-well and cultured at $37{ }^{\circ} \mathrm{C}$ for 1 to 5 days. The medium was replaced, and $100 \mu \mathrm{L}$ RPMI-1640 (containing 10\% CCK-8 solution) was added before the test. After culturing at $37^{\circ} \mathrm{C}$ for $1 \mathrm{~h}$, the absorbances at 450 and $630 \mathrm{~nm}$ were measured. The ratio of optical density (OD) (450-630 nm) values achieved on the following days from the first day (fold change) was calculated to assess the ability of cell proliferation.

\section{Colony formation assay}

For the colony formation assay, 250 cells/well were seeded 
into 6-well plates in triplicate. After ten days of consecutive culture, the cells were fixed with $4 \%$ paraformaldehyde fix solution and stained with $1 \%$ crystal violet solution for $1 \mathrm{~h}$, and clones with more than 50 cells were included in the count.

\section{Transwell migration and invasion assays}

Cells were serum-starved for $24 \mathrm{~h}$ and suspended in serum-free medium. For migration assay, 20,000 cells in a $200 \mu \mathrm{L}$ medium were seeded into the upper chamber of a noncoated $8 \mu \mathrm{m}$-pore-size membrane (Corning, USA) in a 24-well plate, and $600 \mu \mathrm{L}$ complete medium was added to the lower chamber to induce cell migration. After 24-36 h of incubation, cells that adhered to the lower surface of the membrane were fixed with $4 \%$ paraformaldehyde and stained with $1 \%$ crystal violet before observation. For invasion assay, 30,000 cells in $200 \mu \mathrm{L}$ serum-free medium were seeded into the upper chamber of an $8 \mu \mathrm{m}$-pore-size membrane coated with $0.2 \mathrm{mg} / \mathrm{mL}$ Matrigel (BD, USA) in a 24-well plate, and the lower chamber was infused with $600 \mu \mathrm{L}$ of complete medium to induce cell migration. The subsequent steps were similar to Transwell migration assay. Five fields were randomly chosen for cell counting.

\section{Cell cycle analysis}

Cells were collected and washed using phosphate-buffered saline (PBS) three times. The collected cells were fixed in $5 \mathrm{~mL} 70 \%$ ethanol overnight at $-20{ }^{\circ} \mathrm{C}$ and then washed with PBS. After that, cells were resuspended with $400 \mu \mathrm{L}$ of staining solution, containing $20 \mu \mathrm{g} / \mathrm{mL}$ of propidium iodide (PI) and $90 \mu \mathrm{g} / \mathrm{mL}$ of RNase (Sangon Biotech, China) for $30 \mathrm{~min}$ at $37^{\circ} \mathrm{C}$. PI-stained cells were then analyzed using fluorescence-activated cell sorting (FACS; BD). At least 10,000 cells were counted from each sample.

\section{Tumor xenograft animal model}

Male nude mice (4-6 weeks old) were purchased from Slac Laboratory Animals Center (Shanghai, China). Mice were randomly divided into different groups of 7 animals each and fed at the Animal Center of Shanghai Pulmonary Hospital for 1 week before operation. In the operation, $5 \times 10^{6}$ ISOC1 wild-type (WT)/KO A549 cells were subcutaneously injected into the right flanks of nude mice. Mouse xenograft tumors were harvested 5 weeks later, and the tumor volume was calculated using the following formula: (the largest diameter $\times$ the smallest diameter $\left.{ }^{2}\right) / 2(18)$. Experiments were performed under a project license (No. K18-037) granted by Institutional Review Board of Shanghai Pulmonary Hospital, in compliance with institutional guidelines for the care and use of animals.

\section{Co-IP combined with MS assay}

The ISOC1-overexpressing plasmid with flag tag was transfected into the HEK293T cell line. The proteins were harvested using IP lysis buffer (CLB, Beyotime Biotechnology). The proteins were incubated with antiFLAG magnetic beads (Sigma-Aldrich, USA) overnight at $4{ }^{\circ} \mathrm{C}$. The mixture of proteins and beads were washed three times with BC100 buffer (Beyotime Biotechnology). The obtained proteins were separated by $10 \%$ SDS-PAGE, and then detected by MS to identify the proteins interacting with ISOC1.

\section{RNA sequencing}

The total RNA was extracted from A549 cell line of the ISOC1 OE and KO groups, respectively. In preparation for subsequent sequencing, oligo-dT was used to capture mRNA, the mRNA was digested, and the barcodes were obtained. The sequencing was completed using an Illumina Hiseq2500 sequencer (Illumina, USA). The entire sequencing operation was carried out by Shanghai WeHealth BioMedical Technology Co., Ltd. (Shanghai, China). Pathway analysis of differently expressed genes (DEGs) was carried out using the Kyoto Encyclopedia of Genes and Genomes (KEGG) tool (http://www.genome.jp/kegg).

\section{DNA damage detection assay}

A DNA damage quantitation kit (Dojindo) was used to complete the DNA damage detection assay. Cells were treated with cisplatin $(20 \mu \mathrm{M})$ for $2 \mathrm{~h}$ to induce DNA damage, and then gDNA was extracted from cisplatintreated cells using a TIANamp Genomic DNA Kit (Tiangen, China). The number of DNA lesions in each cisplatin-treated sample per 100,000 bases was measured. The experiments were performed according to the manufacturer's instructions.

\section{Bioinformatic analysis}

The mRNA expression levels of ISOC1 in NSCLC and 
normal lung tissues were obtained from UCSC Xena browser (https://xenabrowser.net/) based on the data from The Cancer Genome Atlas lung cancer (TCGA-LUNG) and Genotype-Tissue Expression (GTEx) project. The expression difference between tumor and normal samples was plotted using the R package (http://doi.org/10.5281/ zenodo.2074621). To assess the prognostic significance of ISOC1 in NSCLC, the Gene Expression Profiling Interactive Analysis (GEPIA, http://gepia.cancer-pku.cn/) website was applied to analyze the data of ISOC1 mRNA expression based on 982 samples from the TCGA-LUNG and GTEx project databases.

\section{Statistical analysis}

Data are expressed as mean \pm standard deviation, and independent Student's $t$-tests were employed to compare the differences. Kaplan-Meier analysis was performed to generate survival curves based on the expression level of ISOC1, and log-rank test was carried out to assess the difference of survival curves between the different groups. Multivariate cox regression analysis was used to identify the independent risk factor for survival outcome. Survival curves and hazard ratios (HRs) were used to evaluate the prognostic importance of ISOC1 on disease-free survival (DFS) and OS in NSCLC patients. All statistical analyses were processed by SPSS statistics 17.0 (IBM Corp., USA). A $\mathrm{P}$ value $<0.05$ was considered to be statistically significant.

\section{Results}

ISOC1 was upregulated in NSCLC and was significantly associated with poor DFS

To assess the expression of ISOC1 in NSCLC, a comparison of ISOC1 mRNA expression between 1,122 NSCLC tumor (stage I-IV) and 288 normal lung tissues obtained from the online UCSC Xena database was analyzed. The results showed that mRNA expression of ISOC1 was elevated in NSCLC tissues compared with normal tissues (adjusted $\mathrm{P}<0.001$, Figure S1A). Survival curve analysis showed that higher mRNA expression of ISOC1 was significantly associated with a worse DFS ( $\log$-rank $\mathrm{P}=0.019$; Figure $\mathrm{S} 1 \mathrm{~B})$ but not significantly associated with OS (log-rank $\mathrm{P}=0.72$; Figure $\mathrm{S} 1 \mathrm{C}$ ) in NSCLC patients. Multivariate cox regression analysis showed that elevated ISOC1 expression was an independent risk factor for a decreased DFS in NSCLC patients ( $\mathrm{HR}=1.3, \mathrm{P}=0.020$; Figure $\mathrm{S} 1 \mathrm{~B})$.

\section{ISOC1 promoted NSCLC cell proliferation, migration, and invasion}

To investigate the functions of ISOC1 in lung cancer development, cell proliferation, migration, and invasion assays were carried out in NSCLC cell lines, A549 and H1299. Western blot and qRT-PCR assays were used to assess the protein and mRNA expression of ISOC1 in OE (ISOC1), KD (shISOC1-1 and shISOC1-2), and $\mathrm{KO}$ (ISOC1-KO) groups. The results confirmed that the expression of ISOC1 was successfully elevated in the OE group, downregulated in the $\mathrm{KD}$ group, and absent in the $\mathrm{KO}$ group in A549 (Figure 1A) and H1299 (Figure S2A) cells.

CCK-8 assay was performed to determine the effect of ISOC1 on NSCLC cell proliferation. Forced expression of ISOC1 significantly promoted the proliferation of A549 and H1299 cell lines compared with the control groups, while cell proliferation was significantly decreased in the $\mathrm{KD}$ or KO groups $(\mathrm{P}<0.01$, Figure $1 B$ and Figure $\mathrm{S} 2 \mathrm{~B})$. Transwell assays were carried out to evaluate the migration and invasion ability of ISOC1 OE, KD, and $\mathrm{KO}$ tumor cells. The results of migration assays showed that the ability of NSCLC cell overexpressing ISOC1 to migrate was increased as compared with the control group $(\mathrm{P}<0.05)$. On the other hand, cell migration was restricted when the expression of ISOC1 was blocked $(\mathrm{P}<0.05)$. Consistently, results of invasion assay showed that the number of invading cells in the OE group was increased but was decreased when ISOC1 expression was impaired $(\mathrm{P}<0.05$, Figure $1 C$ and Figure S2C). Furthermore, as shown in Figure $1 D$ and Figure S2D, ISOC1 overexpression promoted NSCLC cell colony formation compared with the control group $(\mathrm{P}<0.01)$, while ISOC1 knockdown inhibited cell colony formation in A549 and H1299 cell lines $(\mathrm{P}<0.05)$.

As ISOC1 expression was elevated in NSCLC, WT and ISOC1 KO A549 cells were used for xenograft tumor growth assays in nude mice to further assess the effect of ISOC1 in the tumorigenesis of NSCLC in vivo. The results showed that ISOC1 deficiency led to a significant decrease of tumor volume compared to WT mice. Hematoxylin-eosin (HE)-stained tumor section confirmed that the xenograft tumor harvested in this study was lung adenocarcinoma $(\mathrm{P}<0.05$, Figure 1E).

\section{miR-4633 functioned as a tumor-promoter in NSCLC}

To determine whether intronic miR-4633 regulates its host 

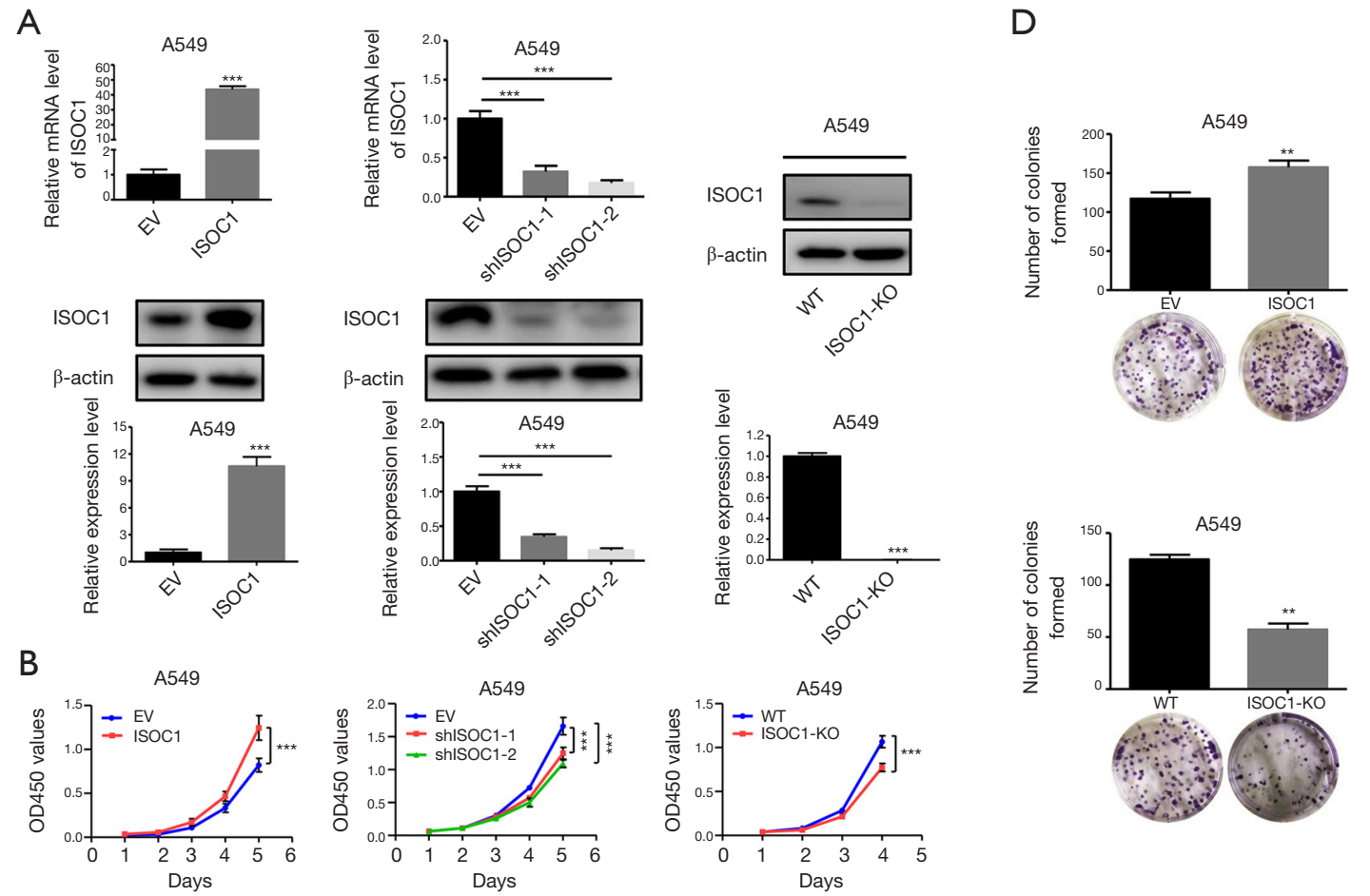

C
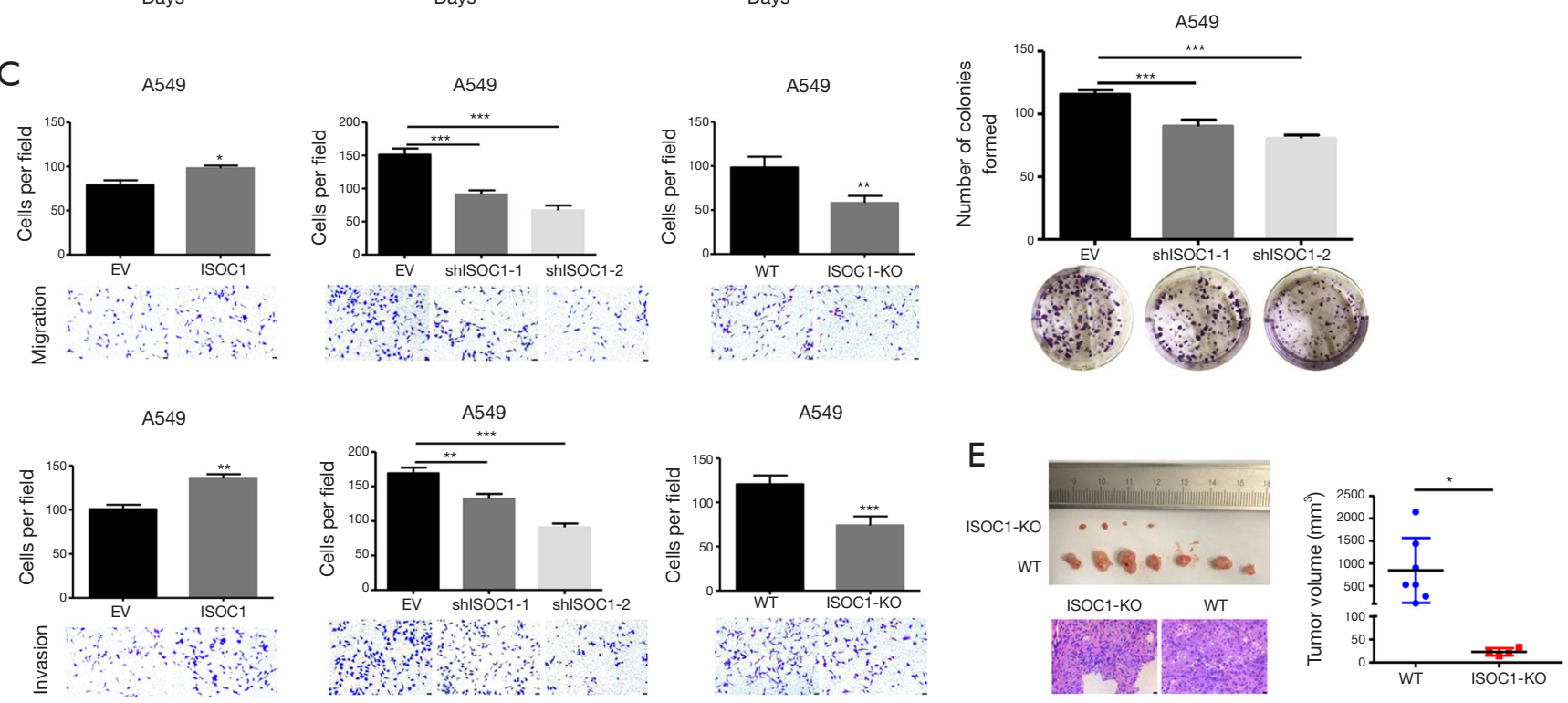

Figure 1 Effects of ISOC1 on NSCLC cell progression, migration, and invasion. (A) OE, KD (shISOC1), and KO efficiency of ISOC1 was determined by Western blot and qRT-PCR. (B) CCK-8 cell proliferation assays in ISOC1 OE, KD, and KO A549 cells from days 1 to 5 . (C) Transwell migration and invasion assays in ISOC1 OE, KD, and KO A549 cells ( $\times 200)$. Cells were stained with crystal violet. (D) Effects of $\mathrm{OE}, \mathrm{KD}$ and $\mathrm{KO}$ of ISOC1 on colony formation in A549 cell line. (E) Tumor xenograft assay performed in the ISOC1 KO group and hematoxylin-eosin staining confirmed tissues as tumorous $(\times 400)$. The number of mice in each group was 7. Three mice in the ISOC1-KO group died 3 weeks after tumor implantation and no suspicious tumor tissues were found subcutaneously. ${ }^{*}, \mathrm{P}<0.05 ;{ }^{* *}, \mathrm{P}<0.01 ;{ }^{* * *}, \mathrm{P}<0.001$. OE, overexpression; KD, knockdown; KO, knockout; EV, empty vector; WT, wild-type. 
A

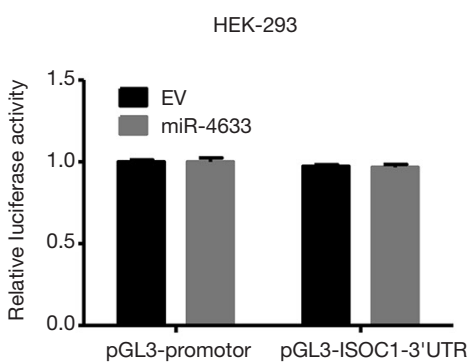

D

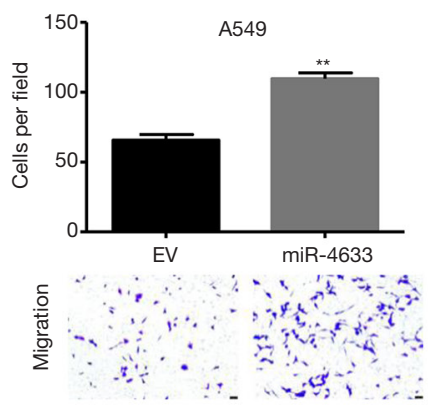

B
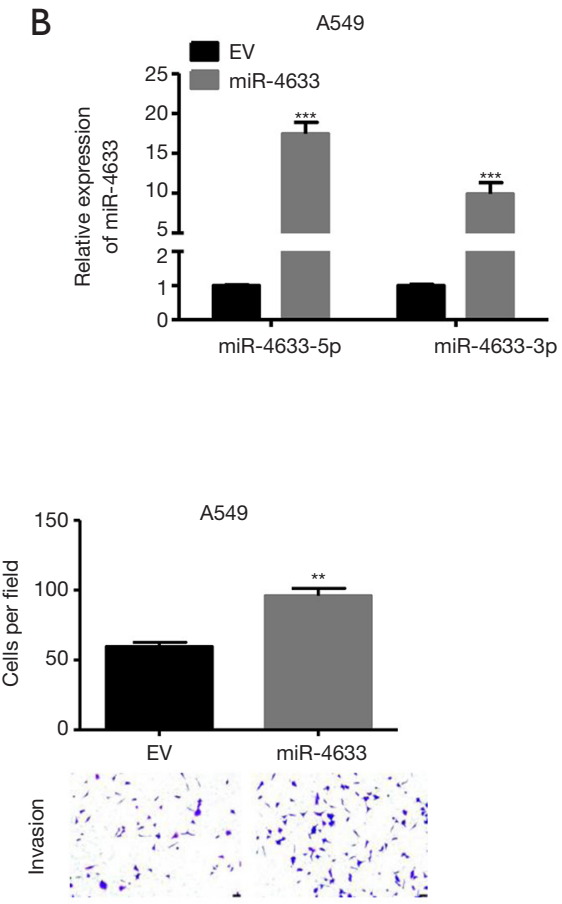

C

A549

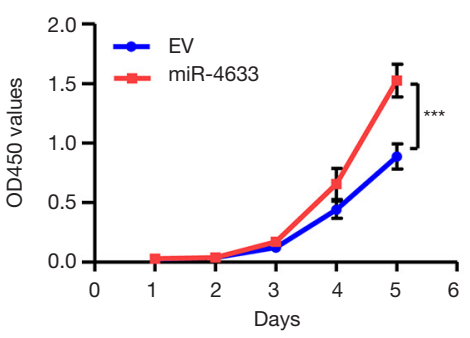

E

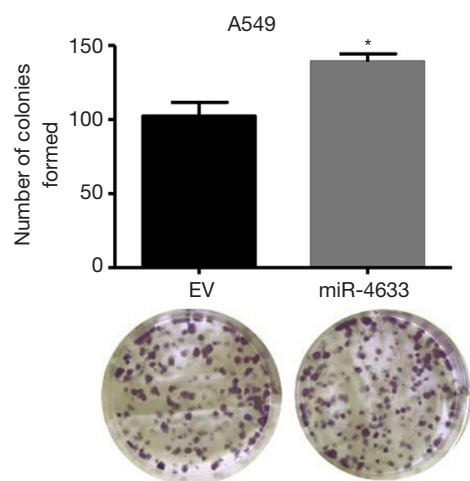

Figure 2 miR-4633 promoted cell viability in NSCLC. (A) Relative luciferase activity of WT ISOC1-3'UTR by miR-4633. (B) Forced expression of miR-4633 in A549 cells was assessed by qRT-PCR. (C) Effects of miR-4633 on A549 cell proliferation was examined by CCK8 assay. (D) Transwell assay was used to assess effects of miR-4633 on A549 migration and invasion ( $\times 200)$. Cells were stained with crystal violet. (E) Colony formation assays in A549 cells infected with EV or miR-4633 lentivirus. *, $\mathrm{P}<0.05$; ${ }^{* *}, \mathrm{P}<0.01$; ${ }^{* * *}, \mathrm{P}<0.001$. WT, wildtype; EV, empty vector.

ISOC1 gene, a dual-luciferase reporter assay was applied in the HEK293 cell line. The results showed that ISOC1 was not the target of miR-4633 ( $\mathrm{P}>0.05$, Figure $2 A)$. As miR4633 showed no apparent effect on regulating its host gene, ISOC1, further investigations on the role of miR-4633 in NSCLC tumorigenesis were performed. The results ensured that the expression of miR-4633 was successfully upregulated in both A549 and $\mathrm{H} 1299$ cell lines $(\mathrm{P}<0.001$, Figure $2 B$ and Figure S3A); furthermore, the overexpression of miR-4633 could promote cell proliferation $(\mathrm{P}<0.001$, Figure $2 C$ and Figure $\mathrm{S3B}$ ), and increase the abilities of cell migration and invasion in A549 and $\mathrm{H} 1299$ cell lines $(\mathrm{P}<0.05$, Figure $2 D$ and Figure $\mathrm{S3C}$ ). The results of colony formation assay also showed that NSCLC cells exhibited an enhanced capacity for colony formation when miR-4633 expression was elevated $(\mathrm{P}<0.05$, Figure $2 E$ and Figure $\mathrm{S} 3 \mathrm{D})$.

\section{ISOC1 and miR-4633 promoted cell cycle progression in NSCLC}

To elucidate the underlying mechanism of ISOC1 and miR-
4633 in promoting NSCLC cell proliferation, cell cycle distribution analysis was performed using flow cytometry. The results showed that the proportion of cells in the $\mathrm{S}$ and G2/M phases was significantly increased in the ISOC1 OE groups, while the proportion of G1-phase cells were significantly decreased $(\mathrm{P}<0.001$, Figure $3 A$ and Figure $\mathrm{S} 4 \mathrm{~A})$; moreover, comparison with the control groups suggested that knockdown of ISOC1 could induce G1 arrest $(\mathrm{P}<0.01$, Figure $3 B$ and Figure S4B). The results of ISOC1 knockout also produced G1 arrest and a decreased proportion of G2/ M-phase cells $(\mathrm{P}<0.05$, Figure $3 C)$. Furthermore, when miR-4633 was overexpressed, a decreased proportion of G1-phase cells and an increased proportion of S-phase cells were observed in both cell lines $(\mathrm{P}<0.05$, Figure $3 D$ and Figure S4C).

\section{ISOC1 interacted with molecules of DNA damage repair and cell cycle arrest patbway}

To investigate the potential mechanism of ISOC1 involved in NSCLC development, CO-IP and MS assay was carried 
A

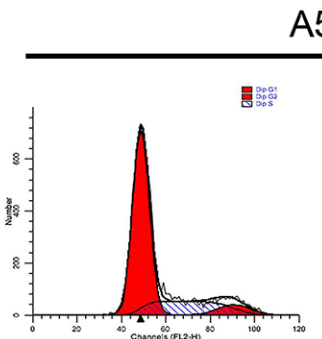

EV

\section{A549}

B

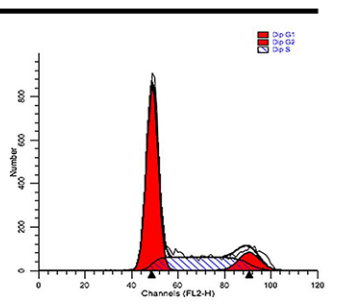

ISOC1

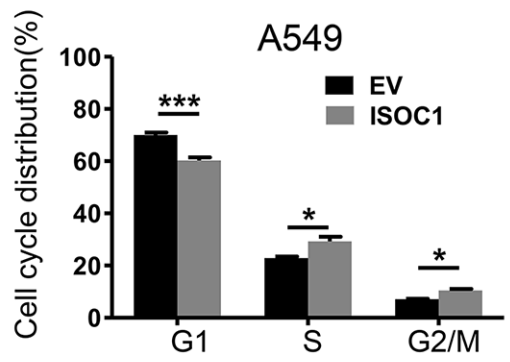

A549

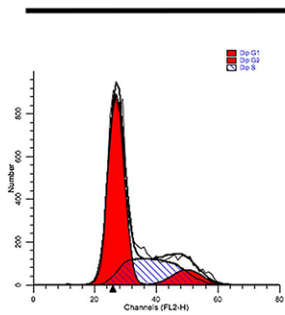

EV

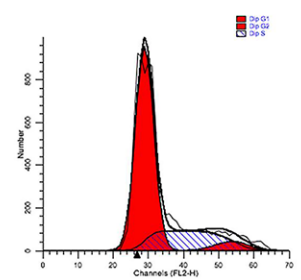

shISOC1-1 A549

C
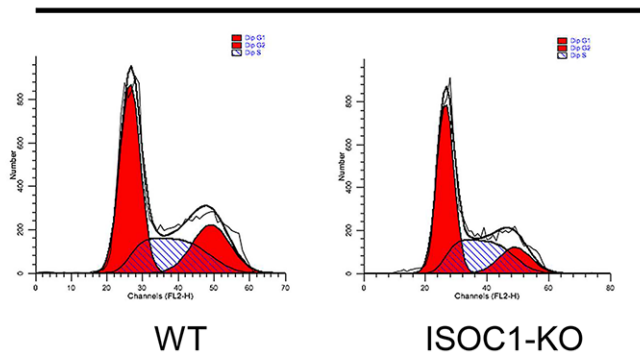

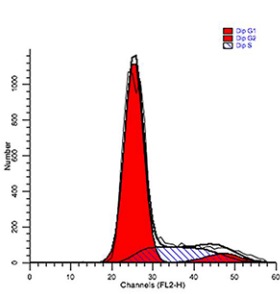

shISOC1-2
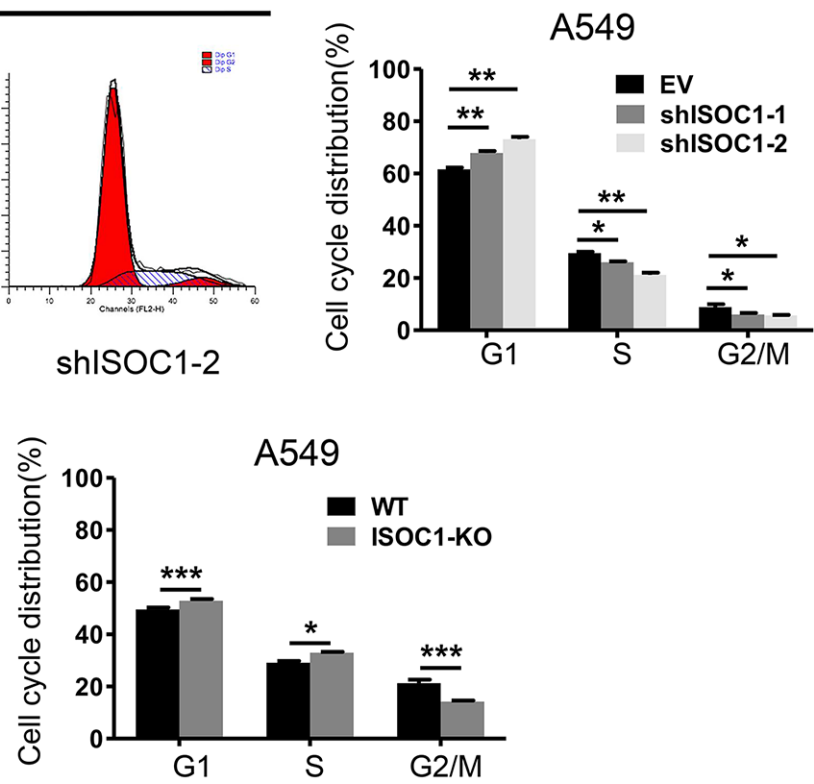

D

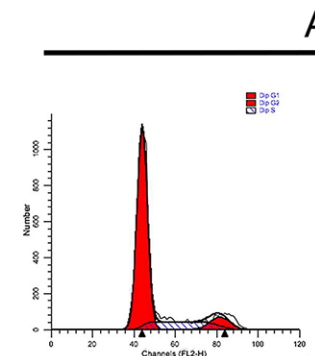

EV
A549

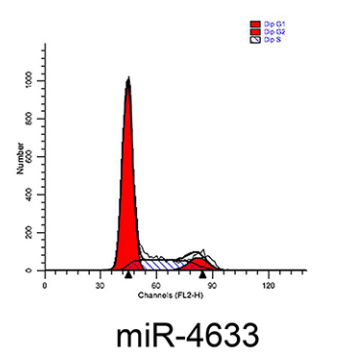

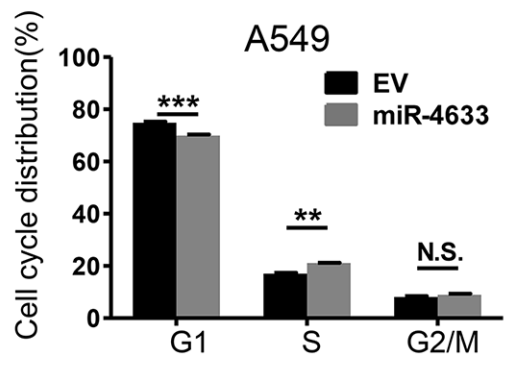

Figure 3 Effects of ISOC1 and miR-4633 on cell cycle distribution. (A) ISOC1 OE in A549 cells caused an increases cell accumulation in S and G2/M phases. (B) ISOC1 knockdown induced G1 arrest in A549 cells. (C) ISOC1 deficiency caused decrease of G2/M phase cells in the A549 cell line. (D) miR-4633 promoted G1/S progression in the A549 cell line. *, $\mathrm{P}<0.05$; ${ }^{* *}, \mathrm{P}<0.01$; ${ }^{* * *}, \mathrm{P}<0.001$. OE, overexpression; KD, knockdown; KO, knockout; EV, empty vector; WT, wild-type; N.S., not significant. 
out to identify the proteins that interact with ISOC1. The results showed that many proteins involved in the DNA damage repair pathway (TP53, PARP1, SFPQ, and NONO) and cell cycle arrest were detected (data not shown). To confirm that ISOC1 participates in DNA damage repair, DNA of cisplatin-treated NSCLC cells was extracted from different groups and detected by DNA damage detection assay. The results showed that overexpression of ISOCI resulted in an increased number of DNA damage lesions $(\mathrm{P}<0.01)$, while the DNA damage sites were decreased when the expression of ISOC1 was downregulated or absent $(\mathrm{P}<0.05$, Figure $4 A)$.

\section{ISOC1 is involved in inflammatory signaling patbway}

To further explore the downstream signaling pathways mediated by ISOC1, RNA sequencing was performed in A549 cell lines of the ISOC1 OE and KO groups. To reveal the potential signaling pathways regulated by ISOC1, KEGG pathway analysis was carried out based on the differential expression of genes in the different groups. The results identified several inflammation-related pathways including IL-17 signaling pathway, TNF signaling pathway, cytokine-cytokine receptor interaction, NF-kappa B signaling pathway, chemokine signaling pathway, and others (Figure 4B,C).

\section{Discussion}

Due to its high morbidity and mortality, lung cancer remains a great threat to human health. The therapeutic landscape of NSCLC has been profoundly expanded by the exploration of specific driver alterations and the use of immune checkpoint inhibitors (19). However, cancer development and progression is a complex process involving a variety of genes and signaling pathways. The efforts to study multiple biomarkers, including their interactions, have contributed to uncovering common targets and enhancing therapeutic efficiency. ISOC1, along with its homolog ISOC2, have not yet been extensively characterized. Previous studies have revealed that ISOC2 may induce cell development by deregulating the activity of tumorsuppressor p16 (INK4a), and may promote tumor growth and differentiation through binding to the inhibitor of histone acetyltransferases and histone deacetylases $(20,21)$. These previous findings suggest that ISOC2 functions as an oncogene in tumorigenesis, and that ISOC1, which belongs to the same family, may play a similar role in tumor progression. Studies have reported that the knockdown of ISOC1 can lead to the upregulation of tumor-suppressive activity in breast cancer, pancreatic cancer, and colon cancer (9-11), but, to the best of our knowledge, few, if any research, has discussed its effect on lung cancer.

To determine the function of ISOC1 in lung cancer development, this study was conducted to clarify the role of ISOC1 in promoting NSCLC development and progression. This study demonstrated that the expression of ISOC1 was significantly increased in malignant lung tumors than normal lung samples, and a higher expression of ISOC1 was associated with worse DFS and a decreased 5-year survival rate. Function investigation showed that elevated expression of ISOC1 could significantly increase cell proliferation and colony formation viability of NSCLC cell lines, and also promote cell migration and invasion in vitro. However, these tumor-promoting phenotypes were suppressed when ISOC1 was silenced. In vivo, xenograft tumors' growth was significantly inhibited when ISOC1 was knocked out using the CRISPR/Cas9 system. These data indicated that ISOC1 exhibited a tumor-promoting function in lung cancer development and progression both in vitro and in vivo. Cell cycle distribution assays illustrated that overexpressing ISOC1 led to a marked increase in the proportion of cells in the $\mathrm{M}$ and $\mathrm{G} 2 / \mathrm{S}$ phases, suggesting that ISOC1 may play an essential role in the regulation of the cell cycle and promote cell cycle progression; while ISOC1 knockdown or knockout induced G1 arrest, constituting an impediment to the G2/M transition protein synthesis and can restrict tumorigenesis in vitro. Co-IP and MS assays revealed that ISOC1 could interact with the DNA damage repair pathway, and further investigation confirmed that overexpression of ISOC1 led to increased DNA damage sites. Dysregulation in the DNA damage repair pathway induces the accumulation of genetic alteration and facilitates carcinogenesis in lung cancer (22). RNA sequencing results showed that the main downstream signaling pathway mediated by ISOC1 was inflammationrelated pathways, including IL-17 signaling pathway, TNF signaling pathway, cytokine-cytokine receptor interaction, NF-kappa B signaling pathway, chemokine signaling pathway, and others. Taken together, these results indicate that ISOC1 interacts with molecules of DNA damage repair pathway and cell cycle arrest to participate in inflammation response. One recent study has shed light on the immune-stimulatory effects of ISOC1 on IL-17 production in memory $\mathrm{T}$ cells (23), and other researches have also implicated the association between ISOC1 and inflammation in neutrophil development and Staphylococcus 
A

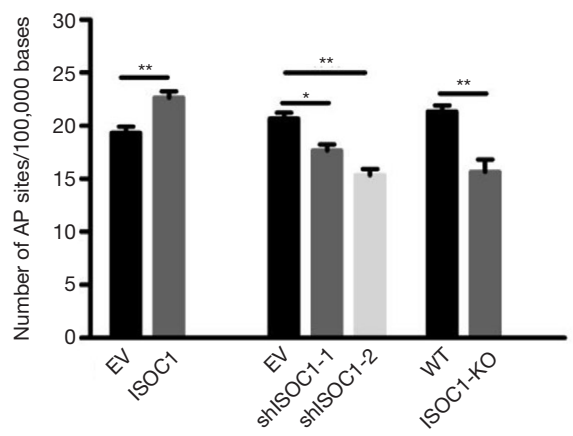

Cisplatin $(20 \mu \mathrm{m})$

B

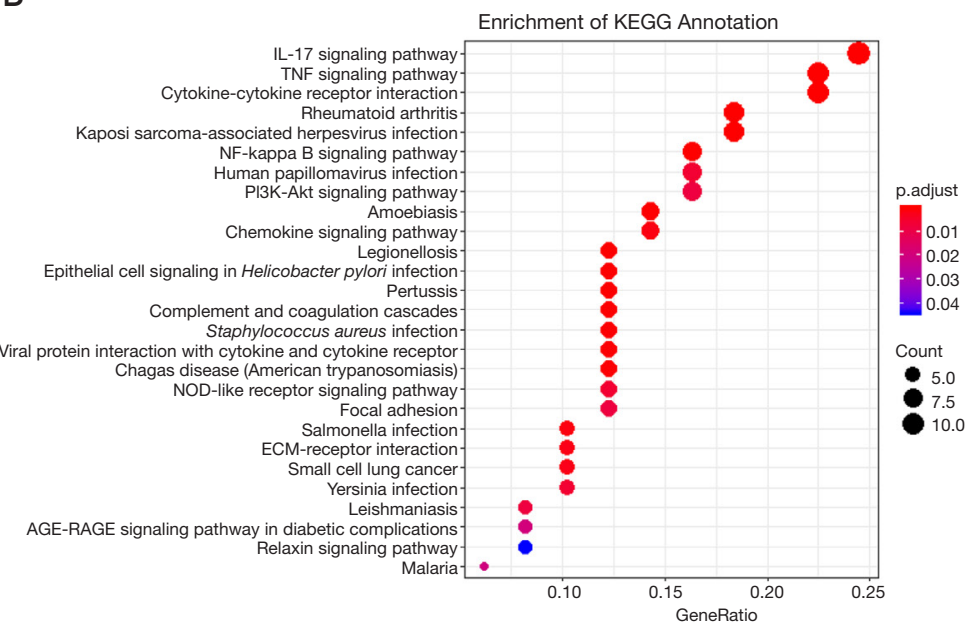

C

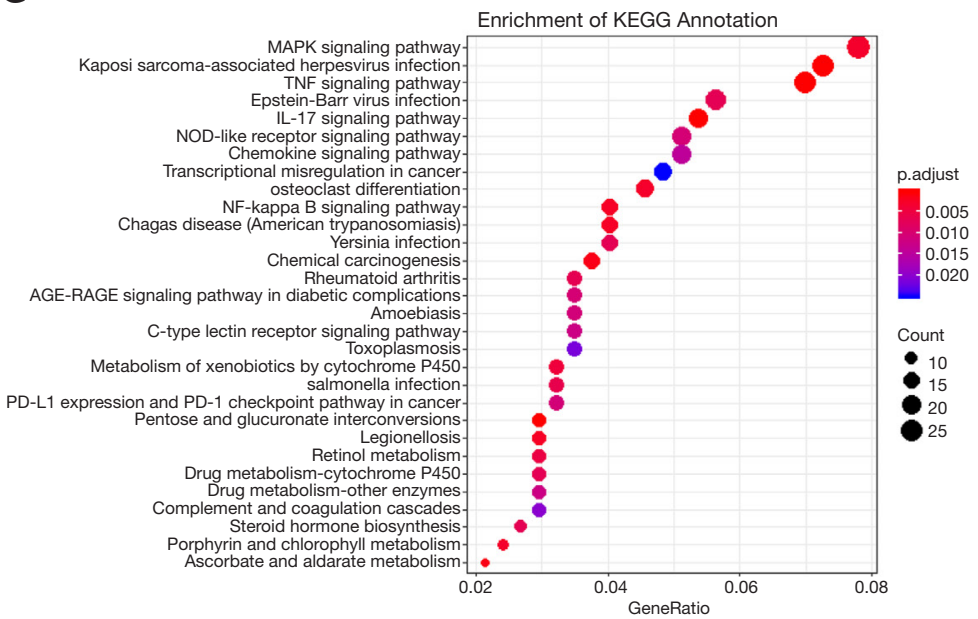

Figure 4 ISOC1 was involved in DNA damage and inflammation-related pathways. (A) Quantitation of the number of DNA damage sites in A549 cells treated with cisplatin $(20 \mu \mathrm{M})$ and detected $2 \mathrm{~h}$ thereafter. (B) KEGG pathway enrichment analysis results based on the RNA sequencing assay in A549 cells with ISOC1 OE. (C) KEGG pathway enrichment analysis results detected by the RNA sequencing assay in A549 cells with ISOC1 KO. *, P<0.05; **, $\mathrm{P}<0.01$; ***, $\mathrm{P}<0.001$. KEGG, Kyoto Encyclopedia of Genes and Genomes; OE, overexpression; $\mathrm{KO}$, knockout. 
aureus infection $(7,24)$, which could further confirm our RNA sequencing data.

Numerous studies have focused on the connection between DNA damage and inflammation, because potential imbalances within the DNA damage repair pathway can promote an inflammatory microenvironment, initiating cancer and facilitating cancer cell growth (25-27). For example, the cGAS-cGAMP-STING pathway mediates the balance between antitumor immunity and inflammationdriven carcinogenesis when DNA damage occurs (28). Our collective findings indicate that ISOC1 is involved in the DNA damage repair pathway and mediates inflammationrelated signaling pathways to promote NSCLC development, and consequently contributes to lung tumorigenesis and cancer cell survival. Therefore, ISOC1 may engage in connecting DNA damage to inflammation and cancer.

A number of miRNAs have been reported to be associated with lung cancer development and behavior (25). Nearly half of miRNAs are classified as intronic miRNAs according to their genomic location (13). It is estimated that approximately $20 \%$ of host genes are targeted by their own intronic miRNAs and are affected by mRNA stability. Previous evidence supports the hypothesis that some miRNAs are co-expressed with their host genes in cancer, acting synergistically or antagonistically $(13,26)$. miR-4633 is encoded in the first intron of ISOC1, and it has been previously studied only in sinonasal mucosal melanoma and lung fibrosis. Here, we found that miR4633 played an important role in NSCLC tumorigenesis itself instead of directly regulating the expression of ISOC1. miR-4633 showed to promote cell proliferation, metastasis, and induce G1-S transition in NSCLC, which indicated that miR-4633 also acted as a tumor-promoting factor in lung cancer development. According to these findings, we hypothesized that miR-4633 and ISOC1 might cooperate to enhance lung cancer development and progression. Zou et al. found that miR-4633 was related to melanoma metastasis, and overexpression of miR-4633-5p, one of the mature forms of miR-4633, inhibited the activation of the PI3K/Akt pathway and its downstream molecule GSK-3 $\beta^{\text {Ser9 }}$ in melanoma cells (14). Surprisingly, Gao et al. recently reported that ISOC1 deficiency could partially activate the Akt/GSK-3 $\beta$ pathway and inhibit cell growth in colon cancer (11). Activating Akt could phosphorylate a serine residue (Ser-9) on GSK-3 $\beta$, which could inhibit kinase activity and ultimately suppress tumor growth (27). These studies indicated that ISOC1 and
miR-4633 might function synergistically through inhibiting the activation of GSK-3 $\beta^{\text {Ser9 }}$, with GSK-3 $\beta$ having been identified as a tumor promoter in most cancer types, including NSCLC (28). More comprehensive and in-depth studies would be needed to assess this relationship in our future work. Our RNA sequencing findings also showed that $I S O C 1$ regulated PI3K/Akt pathway in lung cancer. However, a number of inflammation-related pathways were reported to affect lung cancer patients' prognosis (29), and were revealed to be more significant than PI3K/Akt pathway in NSCLC development mediated by ISOC1 in this study. Further investigation should be performed in the near future.

In conclusion, our present study showed that upregulation of ISOC1 was observed in the NSCLC tumor tissues and was associated with shorter survival. ISOC1 and its intronic miR-4633, both of them could promote NSCLC cell proliferation, migration, invasion, and cell cycle progression. ISOC1 interacts with DNA damage repair pathway and mediates inflammation-related signaling pathways to promote lung tumorigenesis and cancer cell survival.

\section{Acknowledgments}

The authors appreciate the academic support from AME Lung Cancer Collaborative Group.

Funding: This work was supported by the National Natural Science Foundation of China (No. 81802261).

\section{Footnote}

Reporting Checklist: The authors have completed the ARRIVE reporting checklist. Available at http://dx.doi. org/10.21037/tlcr-21-219

Data Sharing Statement: Available at http://dx.doi. org/10.21037/tlcr-21-219

Conflicts of Interest: All authors have completed the ICMJE uniform disclosure form (available at http://dx.doi. org/10.21037/tlcr-21-219). Dr. JM has served on the Advisory Board of Astra Zeneca and Blueprint Medicines. The authors have no other conflicts of interest to declare.

Etbical Statement: The authors are accountable for all aspects of the work in ensuring that questions related to the accuracy or integrity of any part of the work are 
appropriately investigated and resolved. Experiments were performed under a project license (No. K18-037) granted by Institutional Review Board of Shanghai Pulmonary Hospital, in compliance with institutional guidelines for the care and use of animals.

Open Access Statement: This is an Open Access article distributed in accordance with the Creative Commons Attribution-NonCommercial-NoDerivs 4.0 International License (CC BY-NC-ND 4.0), which permits the noncommercial replication and distribution of the article with the strict proviso that no changes or edits are made and the original work is properly cited (including links to both the formal publication through the relevant DOI and the license). See: https://creativecommons.org/licenses/by-nc-nd/4.0/.

\section{References}

1. Planchard D, Popat S, Kerr K, et al. Metastatic non-small cell lung cancer: ESMO Clinical Practice Guidelines for diagnosis, treatment and follow-up. Ann Oncol 2018;29:iv192-iv237.

2. Hoffner B, Leighl NB, Davies M. Toxicity management with combination chemotherapy and programmed death $1 /$ programmed death ligand 1 inhibitor therapy in advanced lung cancer. Cancer Treat Rev 2020;85:101979.

3. Aran V, Omerovic J. Current Approaches in NSCLC Targeting K-RAS and EGFR. Int J Mol Sci 2019;20:5701.

4. Young IG, Gibson F. Regulation of the enzymes involved in the biosynthesis of 2,3-dihydroxybenzoic acid in Aerobacter aerogenes and Escherichia coli. Biochim Biophys Acta 1969;177:401-11.

5. Rainger J, Keighren M, Keene DR, et al. A trans-acting protein effect causes severe eye malformation in the $\mathrm{Mp}$ mouse. PLoS Genet 2013;9:e1003998.

6. Vo TT, Jung EM, Dang VH, et al. Di-(2 ethylhexyl) phthalate and flutamide alter gene expression in the testis of immature male rats. Reprod Biol Endocrinol 2009;7:104.

7. Pedersen CC, Refsgaard JC, Østergaard O, et al. Impact of microRNA-130a on the neutrophil proteome. BMC Immunol 2015;16:70.

8. Litovkin KV, Ivanova OV, Bauer A, et al. Microarray study of gene expression in uterine leiomyoma. Exp Oncol 2008;30:106-11.

9. Yamaga R, Ikeda K, Boele J, et al. Systemic identification of estrogen-regulated genes in breast cancer cells through cap analysis of gene expression mapping. Biochem Biophys
Res Commun 2014;447:531-6.

10. Cheng L, Zhao Y, Tang M, et al. Knockdown of ISOC1 suppresses cell proliferation in pancreatic cancer in vitro. Oncol Lett 2019;17:4263-70.

11. Gao B, Zhao L, Wang F, et al. Knockdown of ISOC1 inhibits the proliferation and migration and induces the apoptosis of colon cancer cells through the AKT/GSK-3 $\beta$ pathway. Carcinogenesis 2020;41:1123-33.

12. Petrek H, Yu AM. MicroRNAs in non-small cell lung cancer: Gene regulation, impact on cancer cellular processes, and therapeutic potential. Pharmacol Res Perspect 2019;7:e00528.

13. Liu B, Shyr Y, Cai J, et al. Interplay between miRNAs and host genes and their role in cancer. Brief Funct Genomics 2018;18:255-66.

14. Zou B, Zhu W, Liu H, et al. Identification and Functional Evaluation of miR-4633-5p as a Biomarker and Tumor Suppressor in Metastatic Melanoma. Cell Physiol Biochem 2018;49:1364-79.

15. Fisher AJ, Cipolla E, Varre A, et al. Potential Mechanisms Underlying TGF- $\beta$-mediated Complement Activation in Lung Fibrosis. Cell Mol Med Open Access 2017;3:14.

16. Lois C, Hong EJ, Pease S, et al. Germline transmission and tissue-specific expression of transgenes delivered by lentiviral vectors. Science 2002;295:868-72.

17. Huai C, Jia C, Sun R, et al. CRISPR/Cas9-mediated somatic and germline gene correction to restore hemostasis in hemophilia B mice. Hum Genet 2017;136:875-83.

18. Naito S, von Eschenbach AC, Giavazzi R, et al. Growth and metastasis of tumor cells isolated from a human renal cell carcinoma implanted into different organs of nude mice. Cancer Res 1986;46:4109-15.

19. Jordan EJ, Kim HR, Arcila ME, et al. Prospective Comprehensive Molecular Characterization of Lung Adenocarcinomas for Efficient Patient Matching to Approved and Emerging Therapies. Cancer Discov 2017;7:596-609.

20. Huang $X$, Shi $Z$, Wang $W$, et al. Identification and characterization of a novel protein ISOC2 that interacts with p16INK4a. Biochem Biophys Res Commun 2007;361:287-93.

21. Fischer JJ, Michaelis S, Schrey AK, et al. SAHA Capture Compound--a novel tool for the profiling of histone deacetylases and the identification of additional vorinostat binders. Proteomics 2011;11:4096-104.

22. Cheng ZY, Hsiao YT, Huang YP, et al. Casticin Induces DNA Damage and Affects DNA Repair Associated Protein Expression in Human Lung Cancer A549 Cells (Running 
Title: Casticin Induces DNA Damage in Lung Cancer Cells). Molecules 2020;25:341.

23. Kushnareva Y, Mathews IT, Andreyev AY, et al. Functional Analysis of Immune Signature Genes in Th1* Memory Cells Links ISOC1 and Pyrimidine Metabolism to IFN-gamma and IL-17 Production. J Immunol 2021;206:1181-93.

24. Ahn SH, Deshmukh H, Johnson N, et al. Two genes on A/J chromosome 18 are associated with susceptibility to Staphylococcus aureus infection by combined microarray and QTL analyses. PLoS Pathog 2010;6:e1001088.

25. Wu KL, Tsai YM, Lien CT, et al. The Roles of MicroRNA in Lung Cancer. Int J Mol Sci 2019;20:1611.

26. Li G, Wu X, Qian W, et al. CCAR1 5' UTR as a natural

Cite this article as: Shi J, Yang F, Zhou N, Jiang Y, Zhao Y, Zhu J, Prelaj A, Malhotra J, Normanno N, Danese E, Cardona AF, Hong X, Jiang G, Song X. Isochorismatase domaincontaining protein 1 (ISOC1) participates in DNA damage repair and inflammation-related pathways to promote lung cancer development. Transl Lung Cancer Res 2021;10(3):14441456. doi: 10.21037/tlcr-21-219
miRancer of miR-1254 overrides tamoxifen resistance.

Cell Res 2016;26:655-73.

27. Beurel E, Grieco SF, Jope RS. Glycogen synthase kinase-3 (GSK3): regulation, actions, and diseases. Pharmacol Ther 2015;148:114-31.

28. O'Flaherty L, Shnyder SD, Cooper PA, et al. Tumor growth suppression using a combination of taxol-based therapy and GSK3 inhibition in non-small cell lung cancer. PLoS One 2019;14:e0214610.

29. Wang XF, Zhu Y'T, Wang JJ, et al. The prognostic value of interleukin-17 in lung cancer: A systematic review with meta-analysis based on Chinese patients. PLoS One 2017;12:e0185168. 
Supplementary

Table S1 Primer sequences used in this study.

\begin{tabular}{|c|c|}
\hline Primer name & Primer sequence (5'-3') \\
\hline ISOC1 forward primer (qPCR) & GAGATTCCCGGAGTCAGGAG \\
\hline ISOC1 reverse primer (qPCR) & TCGTGGTCACTATGATCCCG \\
\hline miR-4633-5p primer (RT) & GTCGTATCCAGTGCGTGTCGTGGAGTCGGCAATTGCACTGGATACGACGAGGAGCT \\
\hline miR-4633-3p primer (RT) & GTCGTATCCAGTGCGTGTCGTGGAGTCGGCAATTGCACTGGATACGACTGCATATG \\
\hline miR-4633 forward primer (qPCR) & CAGTGCGTGTCGTGGAGT \\
\hline miR-4633-5p reverse primer (qPCR) & ACACTCCAGCTGGGATATGCCTGGCTAG \\
\hline miR-4633-3p reverse primer (qPCR) & ACACTCCAGCTGGGAGGAGCTAGCCAGGCA \\
\hline$\beta$-actin forward primer (qPCR) & AGAAGGATTCCTATGTGGGCG \\
\hline$\beta$-actin reverse primer (qPCR) & GGATAGCACAGCCTGGATAGCA \\
\hline U6 forward primer (qPCR) & CTCGCTTCGGCAGCACA \\
\hline U6 reverse primer (qPCR) & AACGCTTCACGAATTTGCGT \\
\hline CD513B-ISOC1 forward primer & TCTAGAGCCACCATGGCGGCTGCGGAGCCGGCGGT \\
\hline CD513B-ISOC1 reverse primer & GGATCCCTACTTATCGTCGTCATCCTTGTAATCTACTTTGGAAAGCAGACCCG \\
\hline CD513B-miR-4633 forward primer & CACAAATGCGTGTGGGAGCGTCACTTACAGCCGAACACAG \\
\hline CD513B-miR-4633 reverse primer & CCACACGCATTTGTGGCGGAGACTTGCCATGTAACCTGCA \\
\hline pLL3.7-shISOC1-1 forward primer & TGTACTTCCAAAGACCAAGTTTTCAAGAGAAAACTTGGTCTTTGGAAGTATTITTC \\
\hline pLL3.7-shISOC1-1 reverse primer & TCGAGAAAAAATACTTCCAAAGACCAAGTTTCTCTTGAAAAACTTGGTCTTTGGAAGTACA \\
\hline pLL3.7-shISOC1-2 forward primer & TGCCTCACAATGTTGTCCACTTATTCAAGAGATAAGTGGACAACATTGTGAGGTTITTC \\
\hline pLL3.7-shISOC1-2 reverse primer & TCGAGAAAAAACCTCACAATGTTGTCCACTTATCTCTTGAATAAGTGGACAACATTGTGAGGCA \\
\hline ISOC1 3'-UTR forward primer & GTGTAATTCTAGACATATGGGGCCCGACATTTGAAGAACTGGTATGCTACTCACTG \\
\hline ISOC1 3'-UTR reverse primer & CCCCGACTCTAGAGAATTCAATTACTTTAATATAAAAGACAGCAGTTTCACATTTCAC \\
\hline ISOC1 sgRNA forward primer & CGACATGCACCGCAAATTCGGTTTAGAGCTAGAAATAGCAAGTTAA \\
\hline ISOC1 sgRNA reverse primer & ACCGAATTTGCGGTGCATGTCGCAAACAAGGCTTTTCTCCAAGG \\
\hline
\end{tabular}



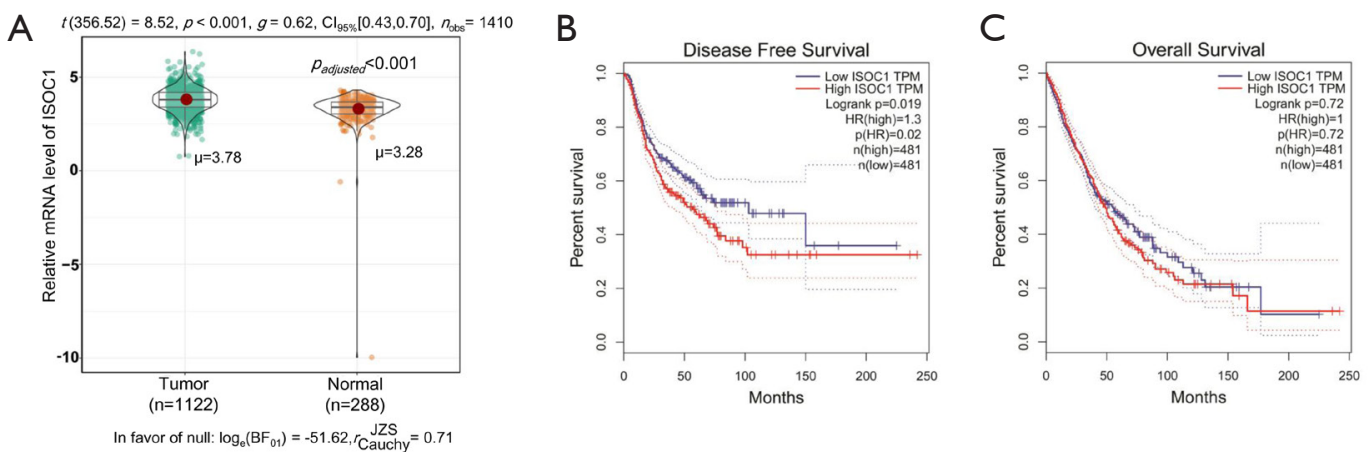

Figure S1 ISOC1 expression was elevated in NSCLC. (A) The expression level of ISOC1 mRNA in NSCLC tumor tissues (n=1,122) and normal lung samples ( $\mathrm{n}=288$ ). Analysis of ISOC1 expression were processed with the data obtained from TCGA and GTEx database using UCSC Xena platform. The horizontal line represents the mRNA expression level $\left[\log _{2}(\mathrm{TPM}+0.001)\right]$. (B) Disease-free survival curve analyses for NSCLC patients based on the expression level of ISOC1 mRNA. (C) Overall survival curve analyses for NSCLC patients based on the expression level of ISOC1 mRNA. TCGA, The Cancer Genome Atlas; GTEx, Genotype-Tissue Expression; TPM, transcripts per million; HR, hazard ratio.

A
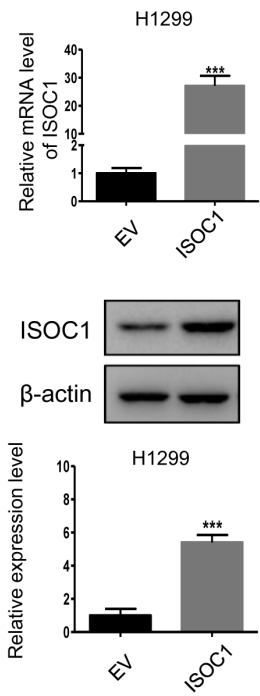

B

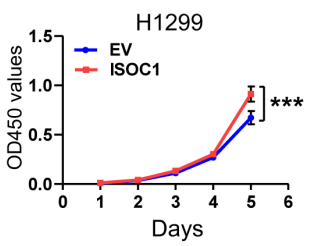

C
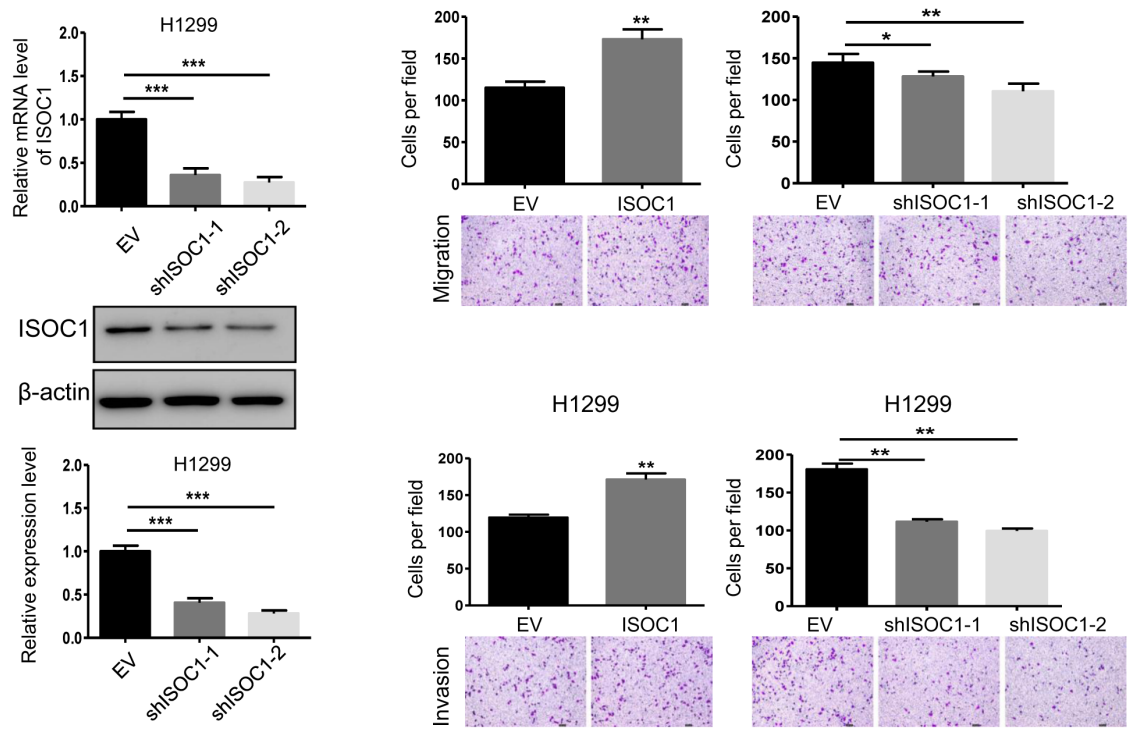

D
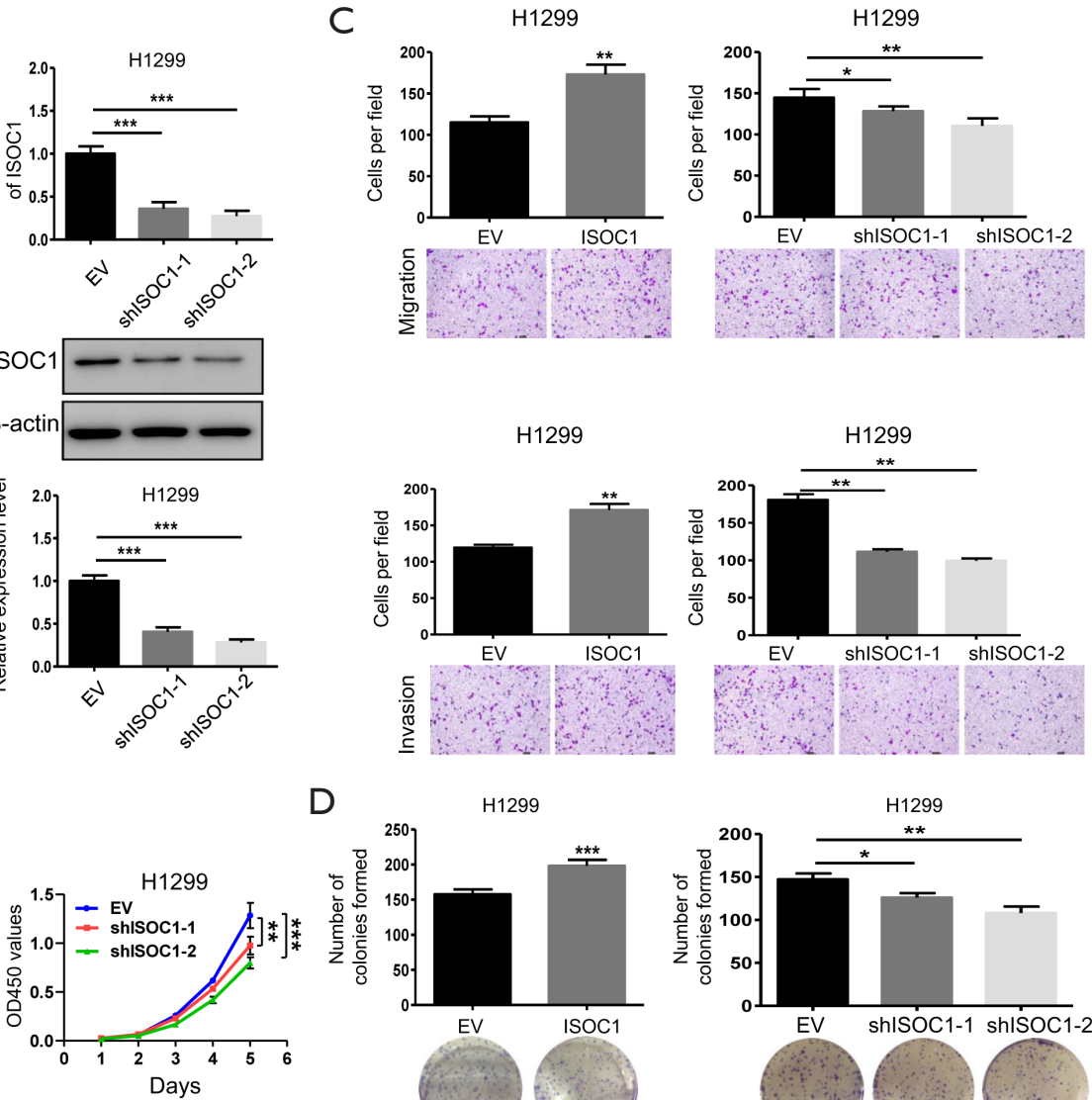

D

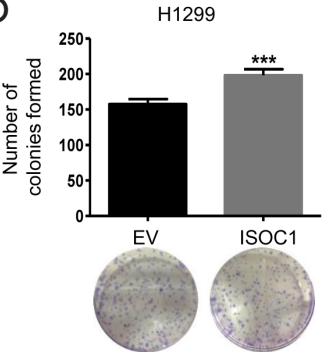

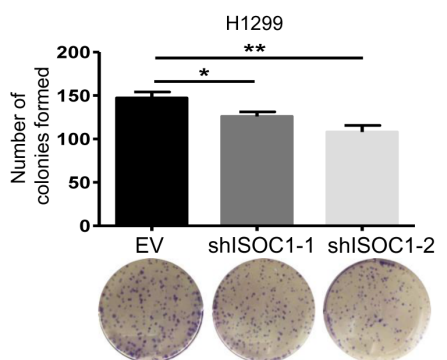

Figure S2 Effects of ISOC1 on H1299 cell progression, migration, and invasion. (A) OE and KD (shISOC1-1 and shISOC1-2) efficiency of ISOC1 was determined by Western blot and qRT-PCR. (B) CCK-8 cell proliferation assays in ISOC1 OE and KD H1299 cells from days 1 to 5. (C) Transwell migration and invasion assays in ISOC1 OE and KD H1299 cells ( $\times 200)$. Cells were stained with crystal violet. (D) Effects of ISOC1 OE and KD on colony formation in the $\mathrm{H} 1299$ cell line. * $\mathrm{P}<0.05$; **, $\mathrm{P}<0.01$; ***, $\mathrm{P}<0.001$. OE, overexpression; KD, knockdown; EV, empty vector. 
A

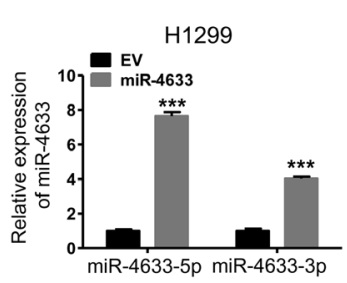

D

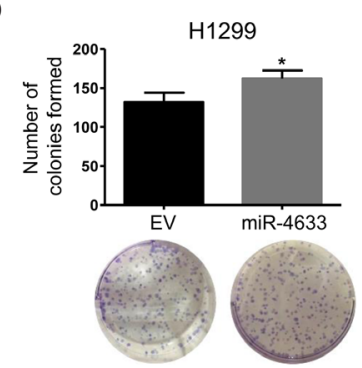

B

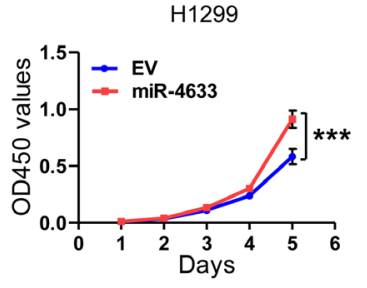

C

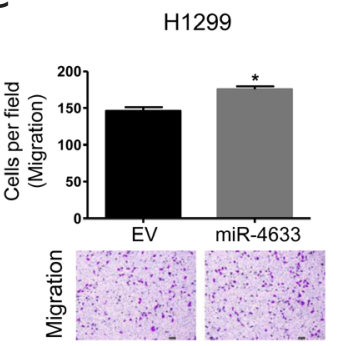

$\mathrm{H} 1299$

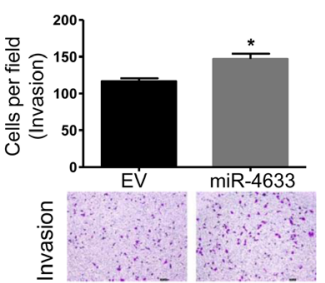

Figure S3 miR-4633 promoted cell viability in the H1299 cell line. (A) Forced expression of miR-4633 in H1299 cells was assessed by qRTPCR. (B) Effects of miR-4633 on H1299 cell proliferation was examined by CCK-8 assay. (C) Transwell assay was used to assess the effects of miR-4633 on H1299 migration and invasion (×200). Cells were stained with crystal violet. (D) Colony formation assays in H1299 cells infected with $\mathrm{EV}$ or miR-4633 lentivirus. * $\mathrm{P}<0.05 ;{ }^{* *}, \mathrm{P}<0.001$. EV, empty vector.

A

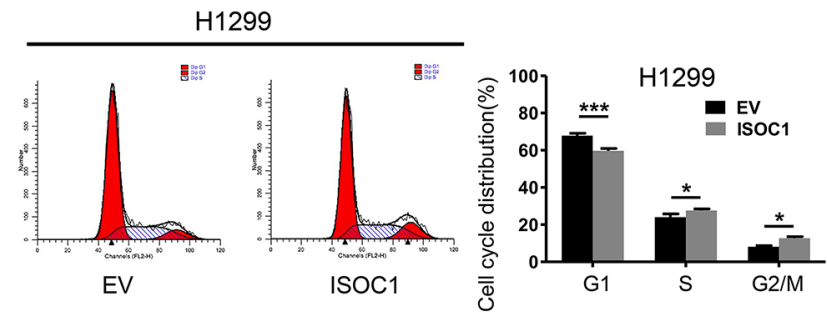

B H1299

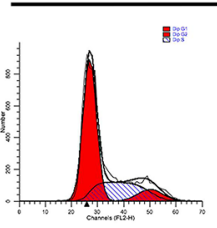

EV

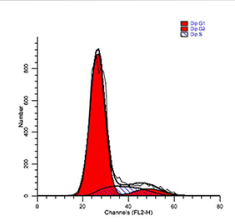

shISOC1-1

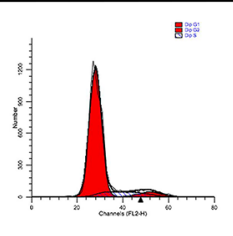

shISOC1-2

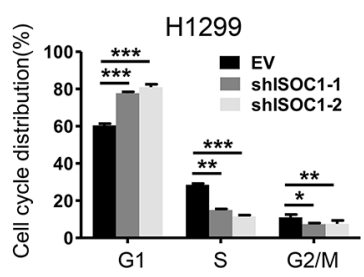

C

$\mathrm{H} 1299$

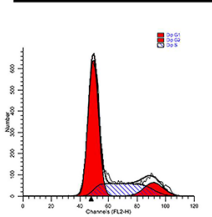

EV

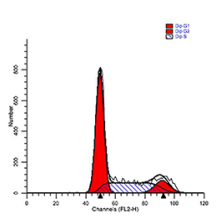

miR-4633

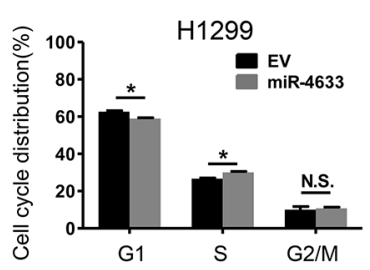

Figure S4 Effects of ISOC1 and miR-4633 on H1299 cell cycle distribution. (A) ISOC1 OE H1299 cells caused an increase of cell accumulation in S and G2/M phases. (B) ISOC1 KD H1299 cell line induced G1 arrest. (C) miR-4633 promoted G1/S progression in the H1299 cell line. * $\mathrm{P}<0.05$; ** $\mathrm{P}<0.01$; ${ }^{* * *}, \mathrm{P}<0.001$. OE, overexpression; KD, knockdown; EV, empty vector; N.S., not significant. 